\title{
Endplate effect on aerodynamic characteristics of three- dimensional wings in close free surface proximity
}

\author{
Jae Hwan Jung ${ }^{1}$, Mi Jeong Kim ${ }^{1}$, Hyun Sik Yoon ${ }^{2}$, Pham Anh Hung ${ }^{1}$ \\ Ho Hwan Chun ${ }^{1}$ and Dong Woo Park ${ }^{3}$ \\ ${ }^{1}$ Department of Naval Architecture and Ocean Engineering, Pusan National University, Busan, Korea \\ ${ }^{2}$ Global Core Research Center for Ships and Offshore Plants, Pusan National University, Busan, Korea \\ ${ }^{3}$ Maritime Research Institute, Hyundai Heavy Industries Co. Ltd, Ulsan, Korea
}

\begin{abstract}
We investigated the aerodynamic characteristics of a three-dimensional (3D) wing with an endplate in the vicinity of the free surface by solving incompressible Navier-Stokes equations with the turbulence closure model. The endplate causes a blockage effect on the flow, and an additional viscous effect especially near the endplate. These combined effects of the endplate significantly reduce the magnitudes of the velocities under the lower surface of the wing, thereby enhancing aerodynamic performance in terms of the force coefficients. The maximum lift-to-drag ratio of a wing with an endplate is increased 46\% compared to that of wing without an endplate at the lowest clearance. The tip vortex of a wing-with-endplate (WWE) moved laterally to a greater extent than that of a wing-without-endplate (WOE). This causes a decrease in the induced drag, resulting in a reduction in the total drag.
\end{abstract}

KEY WORDS: Wing in ground; Endplate; Tip vortex; Lift; Drag.

\section{INTRODUCTION}

The aerodynamic characteristics of a wing-in-ground (WIG) effect craft are of special interest because of the wide applications of the craft during the landing and take-off of airplanes, and for high speed water vehicles. When a wing with small angles of attack operates near the ground, favorable aerodynamic characteristics can be achieved as revealed by early researchers Raymond (1921) and Reid (1927). The lift increases due to a high-pressure air cushion under the wing, and the drag decreases due to the reduction of the downwash velocity (Recant, 1939; Serebrisky and Biachuev, 1946). Consequently, the liftto-drag ratio increases such that the flight range of a WIG vehicle is wider than that of a conventional airplane for a given energy consumption.

Owing to these advantages of WIG vehicles, for several decades, various studies have been performed for 2D and 3D steady/unsteady numerical analysis of WIG by Nuhait and Mook (1989), Mizutani and Suzuki (1993), Hsiun and Chen (1995; 1996), Han et al. (2005), Moon et al. (2005) experimental studies by Fink and Lastinger (1996), Kim et al. (1997), Zerihan and Zhang (2000), Ahmed and Goonaratne (2002), Zhang et al. (2002); the development of a WIG craft by Shin et al. (1997), Chun et al. (1997) the stability analysis by Chun and Chang (2002).

Recently, Ahmed and Sharma (2005) summarized well the experimental investigations into this phenomenon. These studies analyzed the dependence of forces acting on different wing configurations on ground clearance. They also examined the WIG effect on aerodynamic characteristics by experimentally examining the flow around a symmetrical airfoil, NACA 0015, at

Corresponding author: Hyun-Sik Yoon

e-mail:lesmodel@pusan.ac.kr 
angles of attack from $0^{\circ}$ to $10^{\circ}$ for clearance varying from the minimum possible value to one chord length. The studies showed that high values of pressure coefficients $\left(C_{P}\right)$ occur on the lower surface with decreasing ground clearance, leading to high lift when the wing is close to the ground. The results suggest that the pressure distribution on the upper surface does not change significantly regardless of the variation of the ground clearance at higher incident angles.

The other investigative tools used to examine WIG aerodynamic characteristics are the theoretical and numerical methods. However, most theoretical and numerical studies have focused on inviscid flow by using the panel method or lifting surface model. In contrast to inviscid flow, Hsiun and Chen $(1995 ; 1996)$ numerically considered steady 2D WIG effects in laminar viscous flow. They concluded that an airfoil with a larger camber and smaller thickness gave a higher lift-to-drag ratio.

Moon et al. (2005) numerically investigated 3D cambered wing of NACA 4406 with a $2^{\circ}$ angle of attack in ground effect for an aero-levitation electric vehicle at a Reynolds number of $2 \times 10^{6}$ based on the chord length. They solved 3D incomepressible Navier-Stokes equations using the turbulence closure model, and focused on the flow structure according to the configuration of the main and vertical wings by which the high lift-to-drag ratio changes.

Kim et al. (2006) numerically investigated 2D turbulent flow simulations on the low Mach number - high Reynolds number flow about the NACA 4412 airfoil are carried out as the airfoil approaches a ground. It has turned out that angle of attack between $2^{\circ}$ and $8^{\circ}$ is recommended for the airfoil to utilize the benefit of ground effect. For the large angle of attack, the increment of lift due to the ground effect is faded away and negative aerodynamic effect such as destabilizing aspect in static longitudinal stability occurs and the separation point moves to forward as the airfoil approaches a ground.

Recently, authors and coworkers (Jung et al., 2012) numerically investigated the aerodynamic characteristics of 2D wings in the vicinity of the ground by solving 2D steady incompressible Navier-Stokes equations with the turbulence closure model. In addition, they studied the effects of Reynolds number, and showed that the lift-to-drag ratio increases linearly with an increasing Reynolds number.

Although numerous researchers using computational methods have examined the WIG problem, very few computational studies have considered 3D viscous flow and the effect of an endplate on the wingtip over the free surface. Thus, we numerically investigated the aerodynamic characteristics of a 3D wing in the vicinity of the free surface, and the effect of the endplate on the wingtip, by solving incompressible Navier-Stokes equations with the turbulence closure model.

\section{COMPUTATIONAL DETAILS}

The commercial CFD package, Fluent (2009), is employed for all numerical predictions. The present 3D WIG problem is governed by the Navier-Stokes equations and the continuity equation. Once the Reynolds averaging approach for turbulence modeling is applied, the Navier-Stokes equations can be written in Cartesian tensor form as

$$
\begin{gathered}
\frac{\partial u_{i}}{\partial x_{i}}=0 \\
\frac{\partial}{\partial t}\left(\rho u_{i}\right)+\frac{\partial}{\partial x_{j}}\left(\rho u_{i} u_{j}\right)=-\frac{\partial p}{\partial x_{i}}+\frac{\partial}{\partial x_{j}}\left[\mu\left(\frac{\partial u_{i}}{\partial x_{j}}+\frac{\partial u_{j}}{\partial x_{i}}\right)\right]+\frac{\partial}{\partial x_{j}}\left(-\rho \overline{u_{i}^{\prime} u_{j}^{\prime}}\right)+\rho g_{i}
\end{gathered}
$$

where $x_{i}$ are Cartesian coordinates, $u_{i}$ are the corresponding velocity components, $p$ is the pressure, $\rho$ is the density, $\mu$ is the viscosity, and $F_{i}$ is the external body force (e.g. gravity). Also, $-\rho \overline{u_{i}^{\prime} u_{j}^{\prime}}$ in Eq. (2) is the Reynolds stress term, which has been closed by using the SST $k-\omega$ model, because of its improved predictive capabilities compared to the standard $k-\omega$ model, and because of its ability to resolve more accurately and more reliably for a wider class of flow such as the adverse pressure gradient flows and flow around the airfoils. This model ensures that both the near-wall and far-field zones are appropriately solved because an additional cross-diffusion term in the $\omega$ equation was included.

In this study, the volume of fluid (VOF) method is employed to capture the free surface. Most commercial CFD codes use a variation of the VOF approach. In each cell, the volume fraction $\left(Q_{\delta}\right)$ of the $\delta$ th fluid is introduced because this method is 
designed for two or more immiscible fluids. In each cell, the sum of volume fractions of all phases is unity $\left(\sum_{\delta=1}^{n} Q_{\delta}=1\right)$. A cell with a $Q_{\delta}$ value (scalar quantity) of 0 is void and a value of 1 represents a full cell. The cell contains a free surface if the $Q_{\delta}$ value is between 0 and 1 . At each time step, a transport equation is solved to find the distribution of the fluid. At each time step, the distribution of the fluid is found by solving the following transport equation:

$$
\frac{\partial Q_{\delta}}{\partial t}+u_{i} \frac{\partial Q_{\delta}}{\partial x_{i}}=0
$$

A single momentum equation is solved throughout the domain, and the resulting velocity field is shared among the phases (Hirt and Nichols, 1981). Convection and diffusion terms are discretized using the second order upwind scheme and the second order accurate central differencing scheme, respectively. For unsteady flow calculations, time derivative terms are discretized using the first order accurate backward implicit scheme. The velocity-pressure coupling and overall solution procedure are based on a SIMPLE-type segregated algorithm adapted to an unstructured grid. Further details of the implementation can be found in the Fluent (2009) manuals.

Fig. 1 shows a physical model of the 3-D WIG problem, the computational domain, and the coordinate system. A no-slip boundary condition was imposed on the surface of the wing, and a symmetry condition was applied to the far-field boundary. Uniform flow with free-stream velocity $\left(U_{\infty}\right)$ and low turbulence intensity, and a convective condition, were applied to the inflow and outflow boundaries, respectively.

The NACA4406 airfoil under the condition of $\alpha=4^{\circ}$ was selected for the 3-D WIG analysis. The chord length of the wing is represented by $C$. An aspect ratio of $A R=2$ and a Reynolds number $\left(\operatorname{Re}=U_{\infty} C / v\right)$ of $2 \times 10^{6}$ were used.

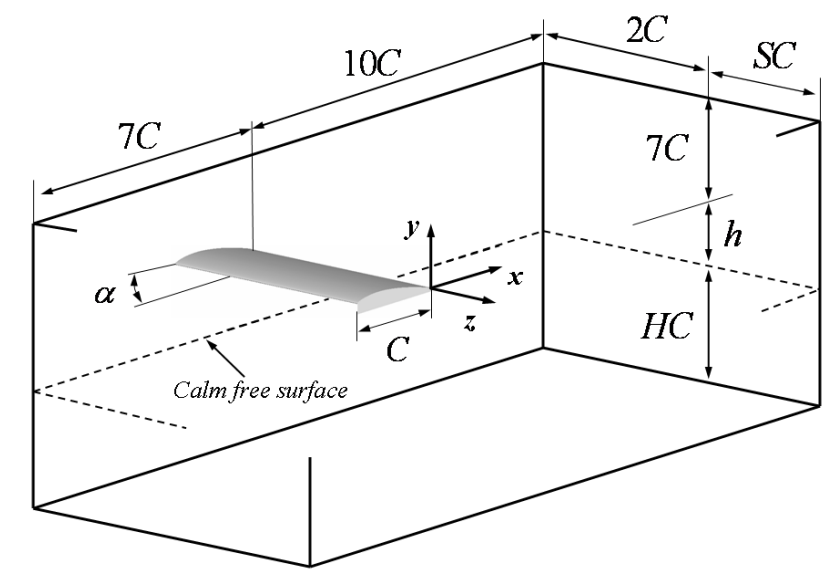

Fig. 1 Physical model of 3-D WIG problem, the computational domain, and the coordinate system.

A free surface was imposed to realize real operational conditions. The normalized clearance between the trailing edge and free surface by the chord length is defined as $h / C$. The effect of an endplate on 3-D WIG behavior was investigated for four different clearances $h / C$ by comparing the bare wing: $h / C=0.05, h / C=0.1, h / C=0.2$ and $h / C=0.3$ for $\alpha=4^{\circ}$ and $\mathrm{Re}=2 \times 10^{6}$. The height of the endplate was fixed at $h / C=0.025$, which was measured from the trailing edge of the wing. However, the thickness of the endplate was neglected to simplify the physical model.

The size of the computational domain was $-7 C \leq x \leq 10 C$ and $-2 C \leq z \leq S C$ for the streamwise and transverse directions, respectively. In the vertical direction, the water and air domains are $-H C \leq y \leq-h$ and $-h \leq y \leq 7 C$. The dependency of the domain size was tested for the WOE case at $h / C=0.1$ for various values of $H$ and $S$. Table 1 shows $C_{L}, C_{D}$, and $f\left(C_{L} / C_{D}\right)$ for different values of $H$ and $S$. Consequently, these values do not strongly depend on $H$ and $S$, which suggests that $H=5$ and $S=6$ are reasonable for the computational domain size of the 3-D WIG problem in terms of computational efficiency. 
Table 1 Dependency of body forces on computational domain size.

\begin{tabular}{|c|c|c|c|c|}
\hline & & $C_{D}$ & $C_{L}$ & $f$ \\
\hline \multirow{3}{*}{$H$} & 2.5 & 0.015167 & 0.387349 & 25.53971 \\
\cline { 2 - 5 } & 5 & 0.015404 & 0.395742 & 25.69012 \\
\cline { 2 - 5 } & 10 & 0.015539 & 0.398841 & 25.66746 \\
\hline \multirow{3}{*}{$S$} & 3 & 0.015541 & 0.398969 & 25.67189 \\
\cline { 2 - 5 } & 6 & 0.015404 & 0.395742 & 25.69012 \\
\cline { 2 - 5 } & 10 & 0.015315 & 0.391894 & 25.58887 \\
\hline
\end{tabular}

Fig. 2 shows a typical grid distribution near the 3-D wing and a calm free surface. To consider the variation of the gap ratio, the number of grid points used in the $y$ direction was tuned to maintain dense resolution near the wing, the free surface, and in the gap spacing. The grids were distributed non-uniformly near these regions, especially in the gap and the wake regions, to capture accurately the separating shear layers on both the wing and the ground. The total number of grids was $2.1 \times 10^{6}$. The minimum vertical grid spacing on the wing was about $10^{-4} C$, corresponding to $y^{+} \approx 2 \sim 4$ as a wall unit, which is similar to the spacing used by Moon et al. (2005) at the same Reynolds number $\left(2 \times 10^{6}\right)$ used in this study.

Validation of the proposed computational method was performed for two 2-D wings, NACA0006 and NACA4406, at $\alpha=2^{\circ}$ and $h / C=0.1$. Fig. 3 shows the distribution of the mean pressure coefficient $\left(\overline{C_{P}}\right)$ on the wing surfaces compared to the results of Moon et al. (2005). The predicted $\overline{C_{P}}$ distributions are in very good agreement with those of Moon et al. (2005).

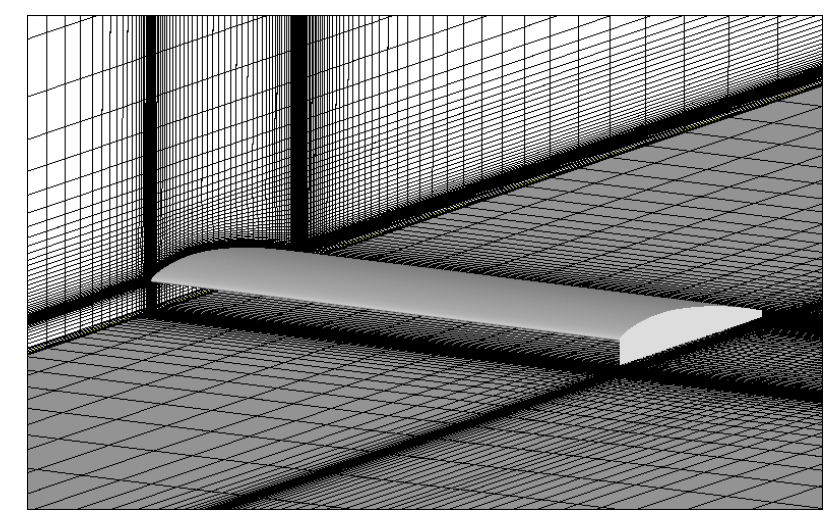

Fig. 2 Typical grid distribution near the 3-D wing, and the calm free surface for $h / C=0.1$.

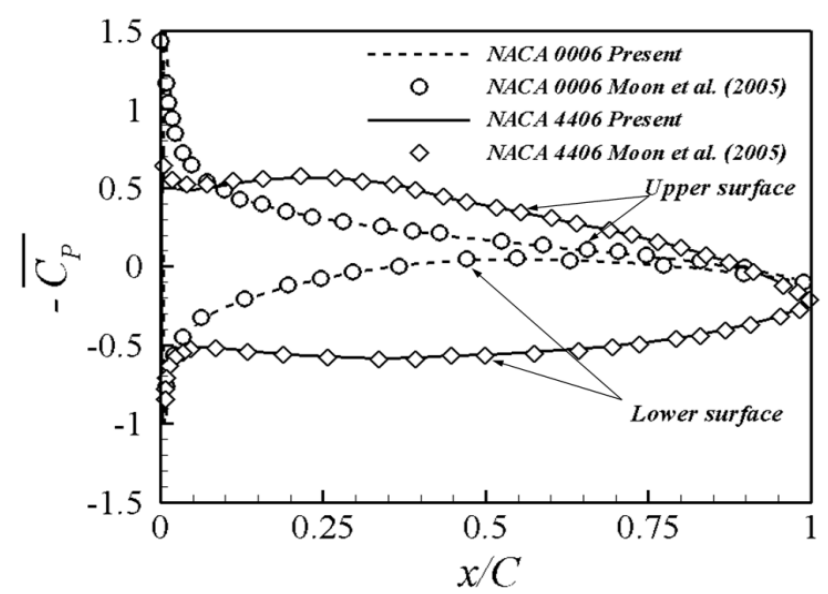

Fig. 3 Distributions of mean pressure coefficient on the surface of NACA0006 and NACA4406 for $\alpha=2^{\circ}$ and $h / C=0.1$. 
For the other verification, a 3-D problem was considered by following the computational conditions used by Moon et al. (2005) for 3-D flow around an isolated NACA0012 of $A R=6$ at $\alpha=5^{\circ}$ and $\mathrm{Re}=5.3 \times 10^{5}$. The total velocity profile across the vortex core at $x / C=5$ of our computation is compared to that of Moon et al. (2005) in Fig. 4. This comparison shows good agreement, and ensures that the grid system is adequate to simulate the wake region.

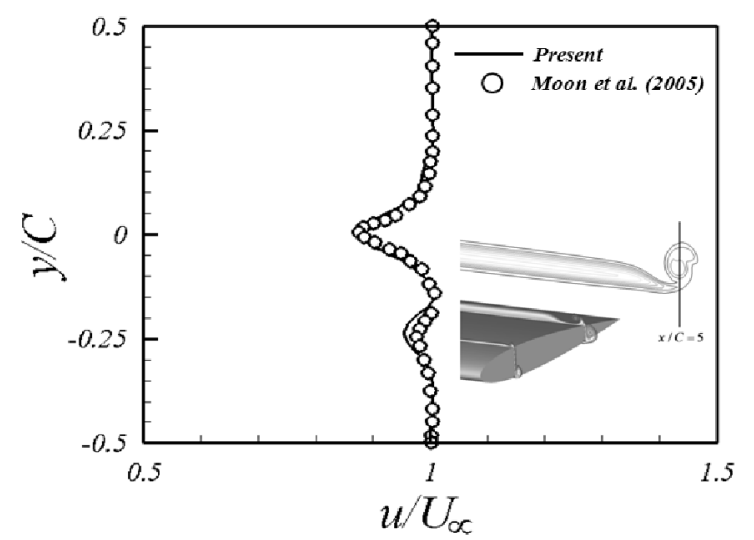

Fig. 4 Total velocity profile across the vortex core at $x / C=5$ in wake.

\section{RESULTS}

Fig. 5 shows the lift $\left(C_{L}\right)$ and $\operatorname{drag}\left(C_{D}\right)$ coefficients and the lift-to-drag ratio $f$ as a function of $h / C$ for a WOE and WWE. Fig. 5(a) shows that the maximum value of $C_{L}$ occurred at the lowest value of $h / C(h / C=0.05)$ among the

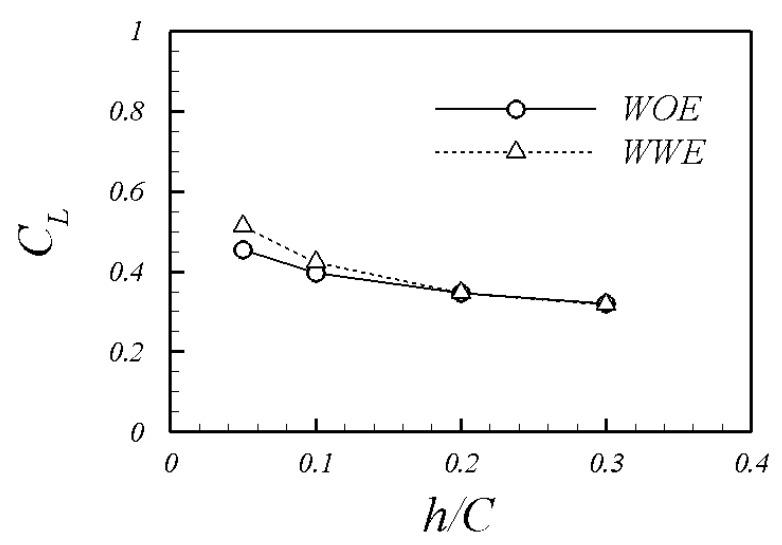

(a)

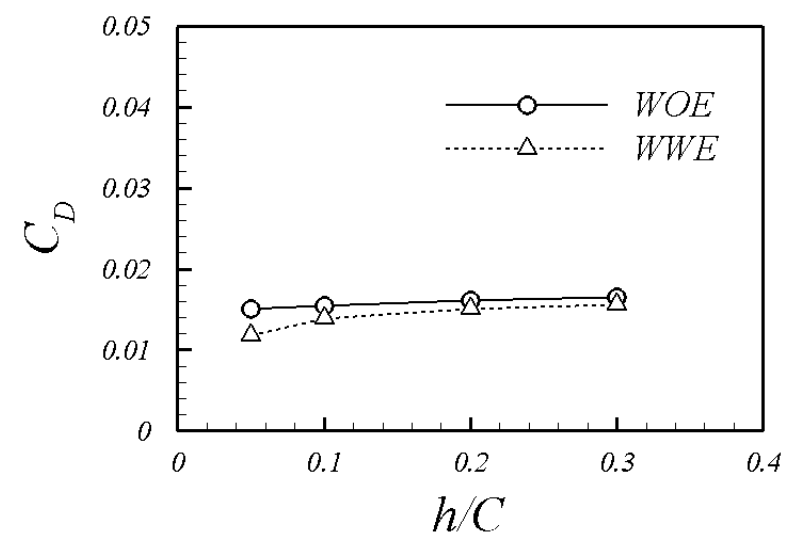

(b)

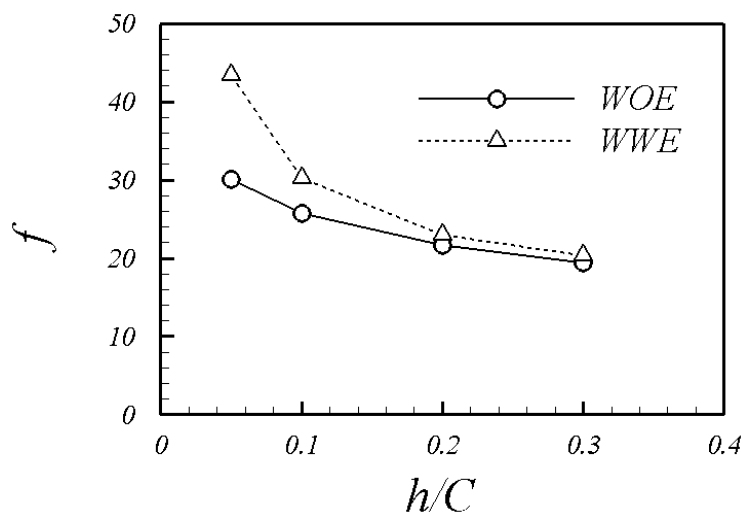

(c)

Fig. 5 Forces along the clearance WOE and WWE. (a) Lift coefficient $C_{L}$. (b) Drag coefficient $C_{D} \cdot$ (c) Lift-to-drag ratio $f$. 
clearances considered in this study for either the WOE or the WWE case. At this clearance, the $C_{L}$ value of WWE is larger than that of WOE. As $h / C$ increased, the $C_{L}$ value of both wings decreased, although the rate of decrease was reduced. When $h / C$ is 0.2 , WOE and WWE have about the same value of $C_{L}$. The drag coefficient of WWE is smaller than that of WOE in the $h / C$ range considered in this study, as shown in Fig. 5(b). In the case of WWE, the increasing rate of $C_{D}$ is distinct for lower $h / C$ ratios. In general, for both wings, as $h / C$ increased, the variation in $C_{D}$ was minimal.

To investigate the aerodynamic performance of WOE and WWE, the $f$ as a function of clearance is shown in Fig. 5(c). The behavior of $f$ versus clearance can be estimated from $C_{D}$ and $C_{L}$ as shown in Figs. 5(a) and (b), respectively. The highest $f$ value appears at the lowest $h / C$ of 0.05 , independent of the endplate. The maximum $f$ value of WWE increased by $46 \%$ compared to that of WOE. The difference in $f$ between WOE and WWE decreased with increasing $h / C$, resulting in both wings producing approximately the same value of $f$ at the largest value of $h / C=0.03$.

Fig. 6 shows the contours of $\overline{C_{P}}$ on the lower and upper surfaces for WOE and WWE at two different clearances of $h / C=0.05$ and $h / C=0.2$. At $h / C=0.05$, where the difference in aerodynamic performance between WOE and WWE is the largest as shown in Figs. 5(a-c), the case of WWE presents larger absolute values of negative and positive $C_{P}$ on the suction side (upper) surface and the pressure side (lower) surface than in the case of WOE. This can be clarified by comparing
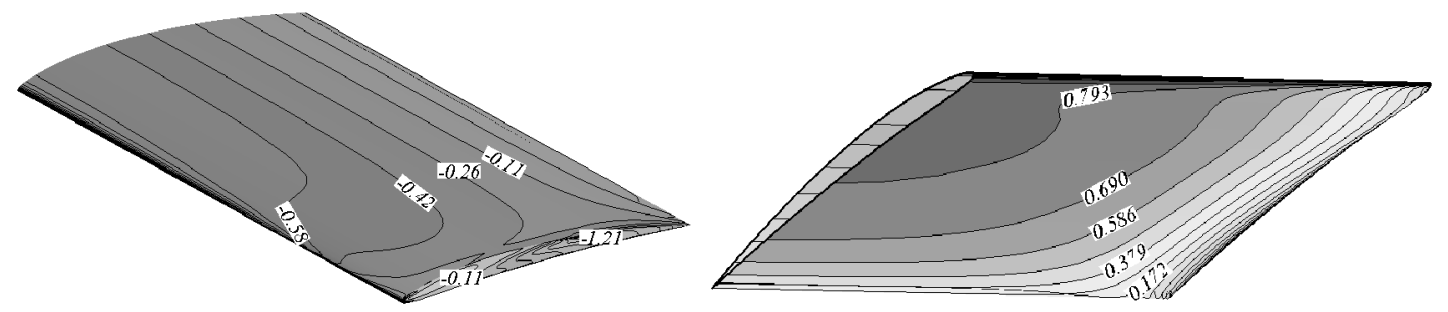

(a) WOE at $h / C=0.05$.
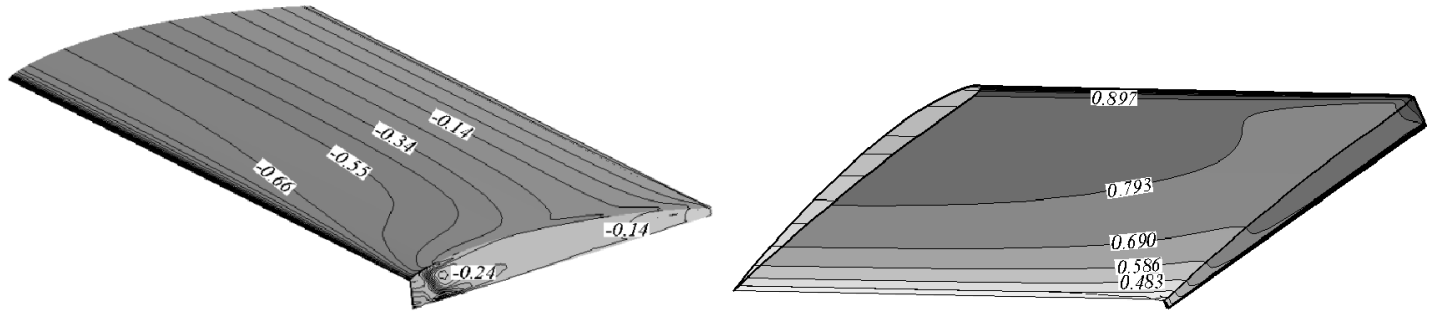

(b) WWE at $h / C=0.05$.
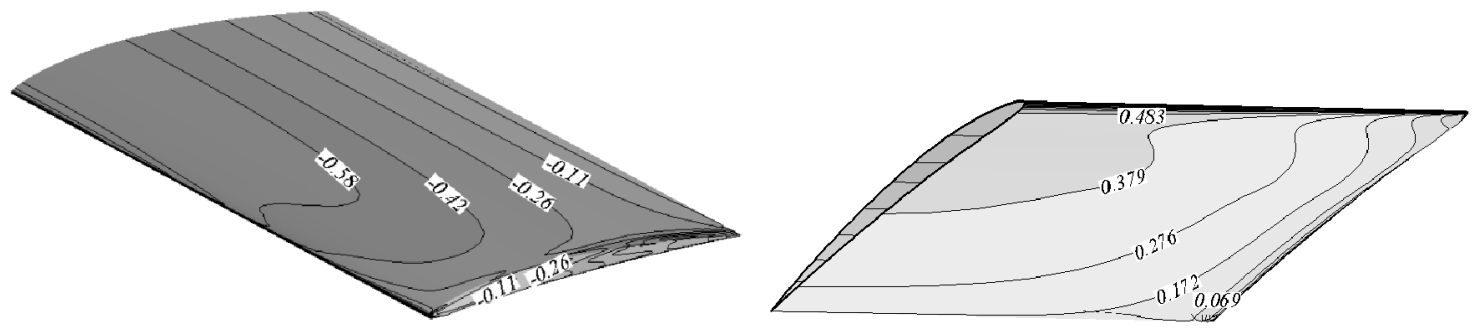

(c) WOE at $h / C=0.2$.
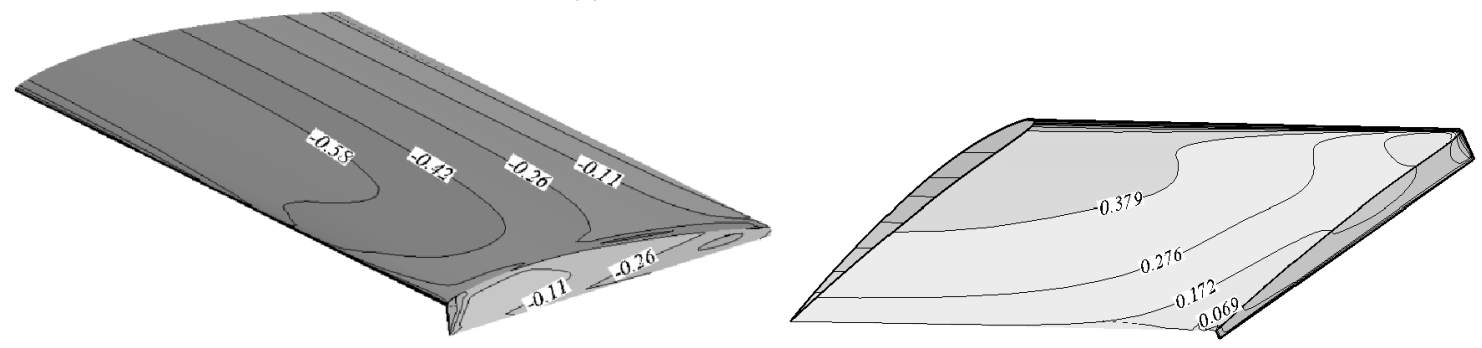

(d) WWE at $h / C=0.2$.

Fig. 6 Mean pressure coefficient distribution on upper (left column) and lower (right column) surfaces for (a) WOE, (b) WWE at $h / C=0.05$, (c) WOE, and (d) WWE at $h / C=0.2$. 
Fig. 6(a) for WOE with Fig. 6(b) for WWE. Especially for the lower surface, the endplate enhanced the air cushion by blockage of the flow between the endplates. As a result, the $C_{P}$ increases $14 \%$ and $124 \%$ near the leading edge and the trailing edge, respectively, compared to the WOE case. Also, the endplate contributes to extending the high $C_{P}$ toward the wingtip.

As $h / C$ increased to 0.2 , the pressure distribution on both surfaces of WOE was very similar to that of WWE as shown in Figs. 6(c) and (d), respectively, since the endplate effect weakened with increasing $h / C$. This validates the finding that the difference in the force coefficients between WOE and WWE is negligible at large values of $h / C$, as shown in Fig. 5.

Fig. 7 shows the variation of $\overline{C_{P}}$ on the airfoil surface at the location of $75 \%$ wing span for WOE and WWE at three different clearances: $h / C=0.05, h / C=0.1$ and $h / C=0.2$. Fig. 7 shows that the effect of the endplate is significant at low clearance, which is validated by the pressure contour distribution shown in Figs. 6(a) and (b) for WOE and WWE, respectively. In particular, $\overline{C_{P}}$ over the pressure side (lower surface) of $h / C=0.05$ is much larger than the values of $\overline{C_{P}}$ of other Cases, resulting in the largest $C_{L}$.

In order to observe the effect of the endplate on the magnitude of the velocity, which is directly related to the pressure variation, the $\mathrm{u}$ and $\mathrm{w}$ velocity contours at $75 \%$ of the wing span are shown in Fig. 8 . These velocity contours correspond to the profiles of the $\overline{C_{P}}$ on the wing's surface as shown in Fig. 7. Since the dependence of the normal velocity $v$ on the endplate compared to $u$ and $w$ is relatively weak, the $v$ velocity is not considered here for brevity.

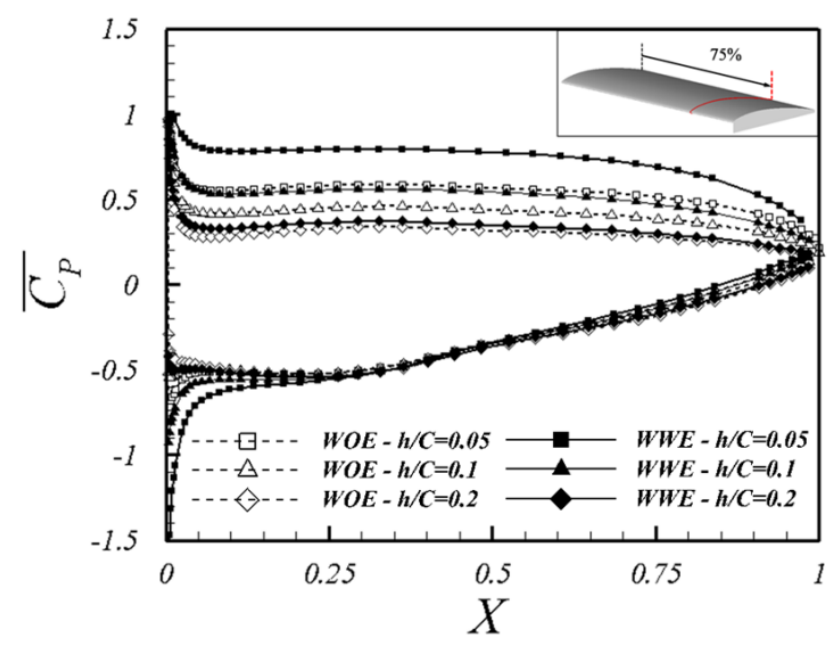

Fig. 7 Mean pressure coefficient distribution at $75 \%$ of wingspan.

We suggest that the endplate causes the blockage effect on the flow and the additional viscous effect, especially near the endplate. This combined effect of the endplate significantly reduces the magnitude of the $u$ and $w$ velocity components under the lower surface of the wing, thereby enhancing aerodynamic performance in terms of the force coefficients as confirmed by Figs. 5 7. The largest combined effect of the endplate occurred at the lowest clearance value of $h / C=0.05$, which can be illustrated by comparing each velocity of WOE to that of WWE in Figs. 8(a) and (b).

As $h / C$ increases, the magnitude of both velocity components increases due to the weakness of the wing-in-ground effect. In addition, the effect of the endplate decreases with increasing $h / C$. Thus, at $h / C=0.2$, WOE and WWE have about the same $u$ and $w$ velocity magnitude values, as shown in Figs. 8(c) and (d).

The formation of the tip vortex for WOE and WWE is plotted in Fig. 9 by using the streamlines in the $y-z$ plane at $h / C=0.05$. At $x=-0.75$ near the leading edge, WOE formed small two vortices on the side and upper surfaces by flow separated from the edges of the wing tip, as shown in Fig. 9(a). In contrast to WOE, WWE formed a relatively larger vortex near the tip of the endplate by flow separation. The primary vortex of the wing tip for WOE evolved along the downstream direction up to the trailing edge, as attached to the wing tip, as shown in Figs. 9(a) (c). However, in the case of WWE, the primary vortex originated from the endplate tip and moved more laterally in comparison to that of WOE. WWE formed the secondary vortex earlier over the free surface because the large primary vortex initiated from the endplate tip formed an accelerated gap flow that separated from the free surface. Farther downstream, the vortices of both wings increased in size and slightly deformed the free surface, as shown in Fig. 9(d). 

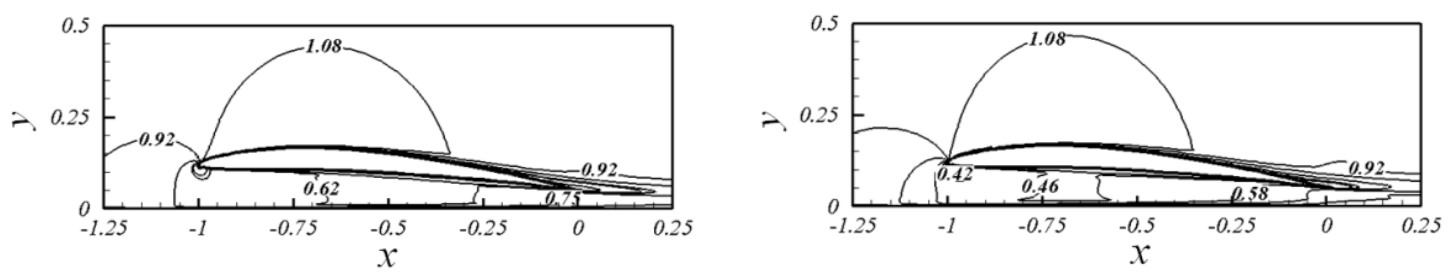

(a)
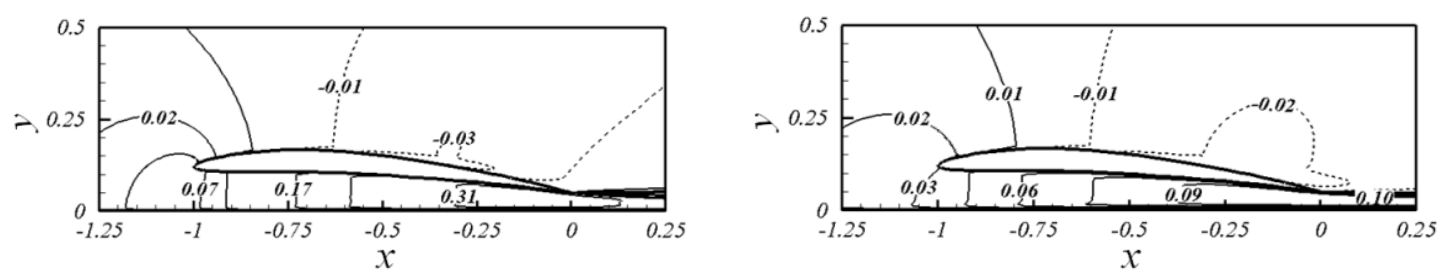

(b)
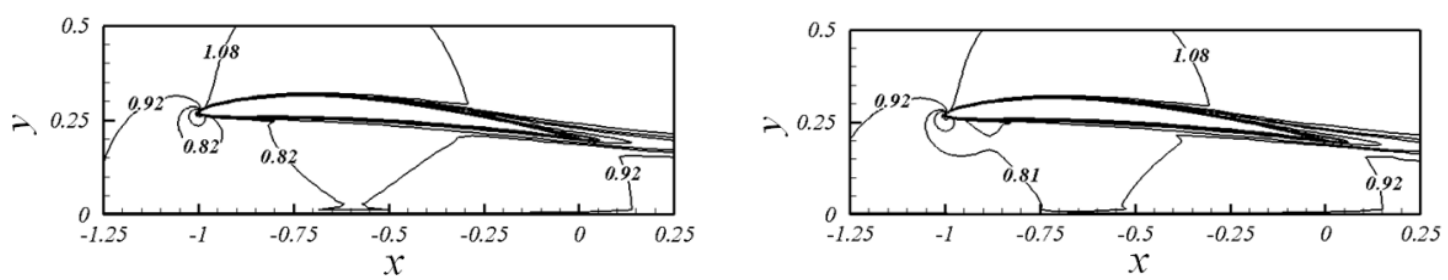

(c)
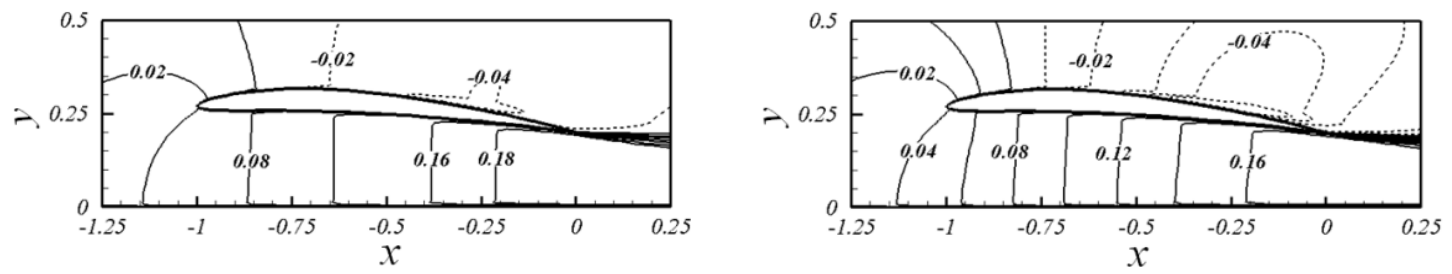

(d)

Fig. 8 Contours of (a) $u$ and (b) $w$ velocities at $h / C=0.05$, and (c) $u$ and (d) $w$ velocities at $h / C=0.2$ for WOE (left column) and WWE (right column) at $75 \%$ of wingspan.
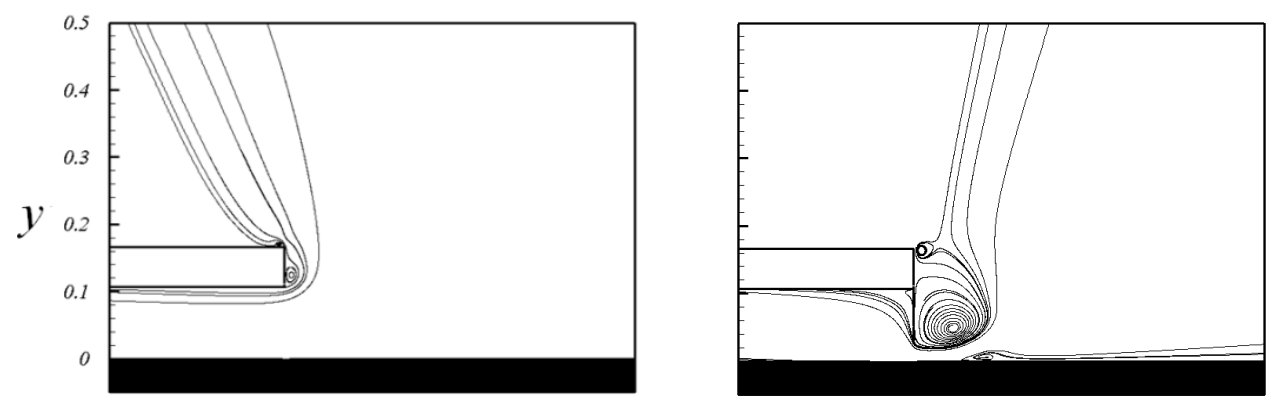

(a)
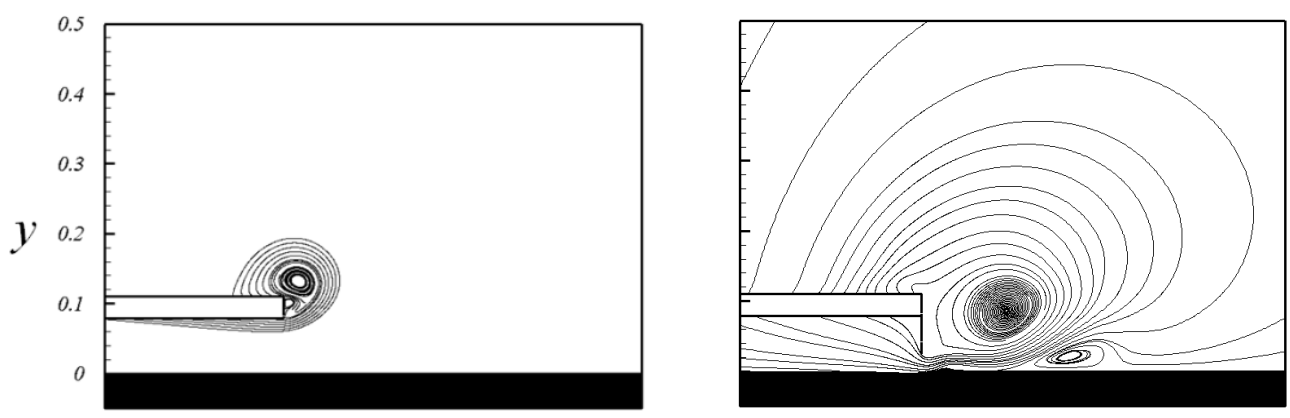

(b) 

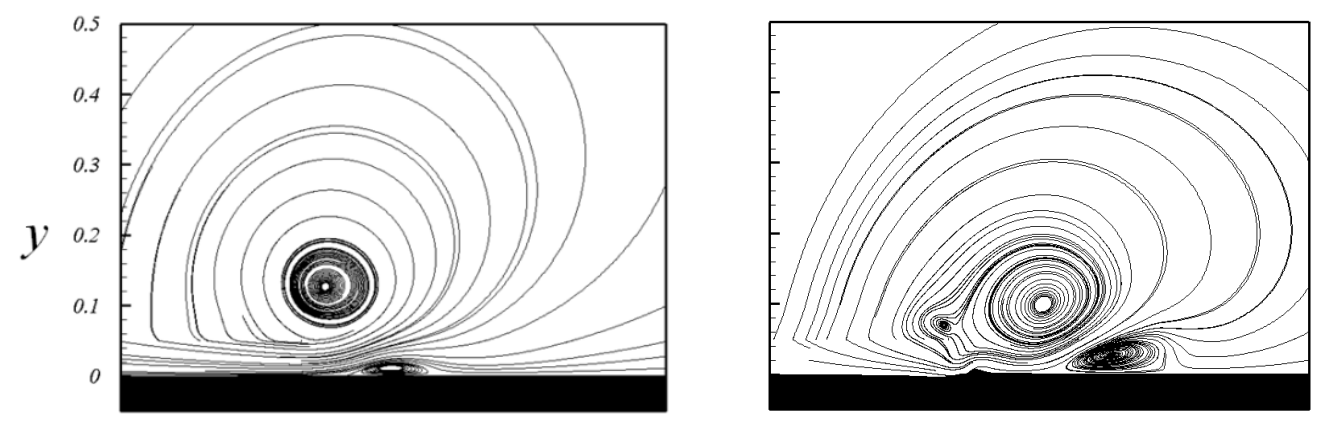

(c)
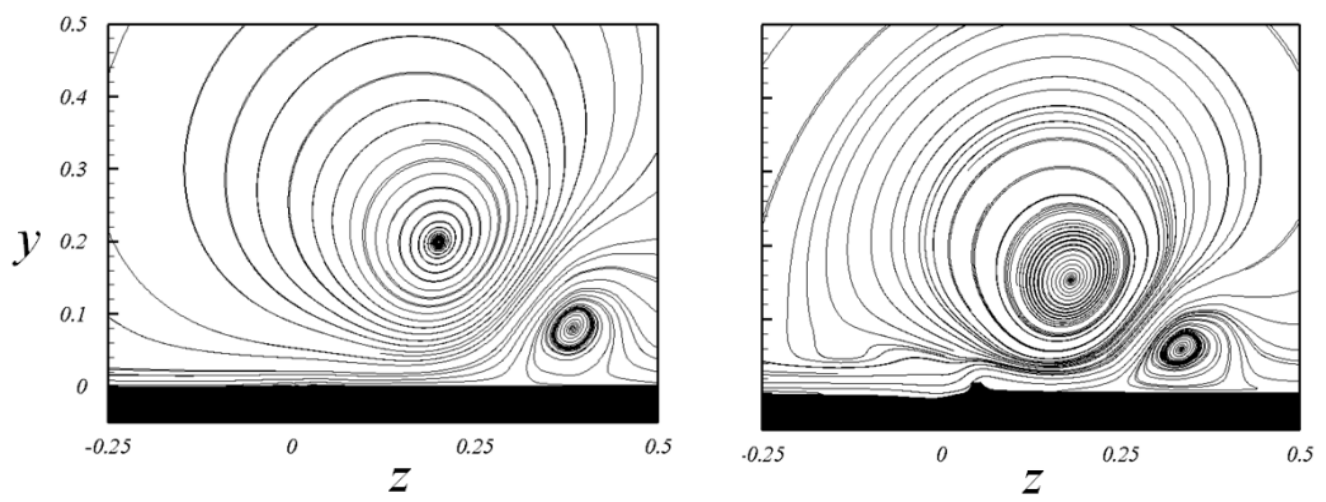

(d)

Fig. 9 Streamlines in $y-z$ plane at $h / C=0.05$ for WOE (left column) and WWE (right column) at different streamwise locations of (a) $x=-0.75$, (b) $x=-0.25$, (c) $x=0$, and (d) $x=1$.

Fig. 10 depicts the trajectories of vortices by extracting the center of each vortex shown in Fig. 9. The endplate of WWE generates the primary vortex (V1) and secondary vortex (V2) from almost the leading edge. However, the V2 of WOE forms from the trailing edge, which dissipates earlier than that of WWE. The V1 of WWE moves more laterally between the leading and trailing edges. This large lateral movement of the V1 of WWE ensures sufficient spacing near the trailing edge to develop the tertiary vortex (V3), which forms from the endplate edge.

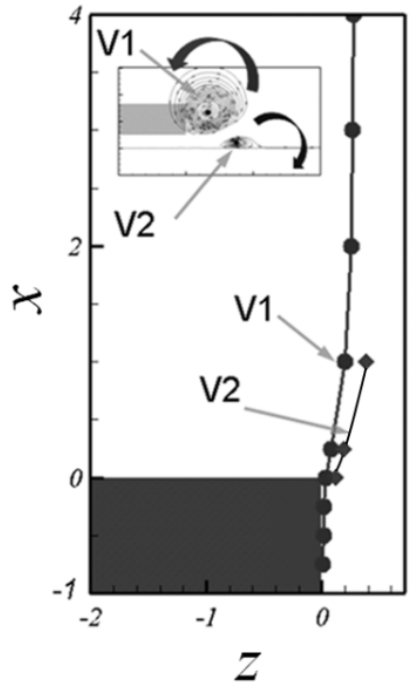

(a)

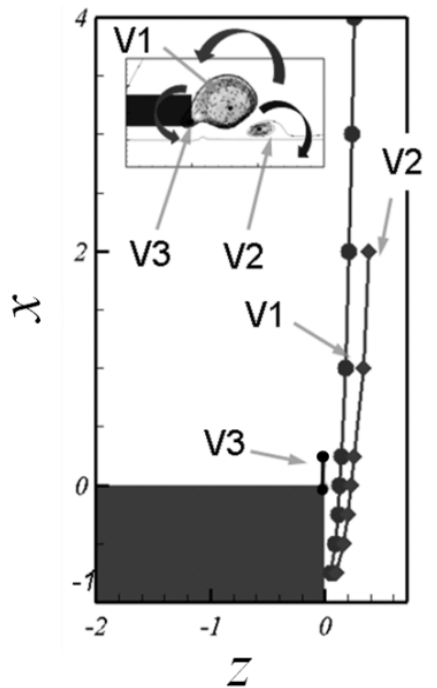

(b)

Fig. 10 Trajectories of tip vortices in $x-z$ plane for (a) WOE (left column) and

(b) WWE (right column) at $h / C=0.05$. 


\section{CONCLUSIONS}

We numerically investigated the effect of an endplate on the 3-D WIG problem using the assumptions of steady incomepressible viscous flow. The NACA4406 airfoil with $A R=2$ at $\alpha=4^{\circ}$ was considered for $\operatorname{Re}=2 \times 10^{6}$. A free surface was imposed to realize the real operational conditions. Four different clearances $(h / C)$ for a fixed height of the endplate were considered in order to analyze the gap effect.

The endplate causes a blockage effect on the flow and an additional viscous effect, especially near the endplate. This combined effect of the endplate significantly reduces the magnitudes of the velocities under the lower surface of the wing. The largest combined effect of the endplate occurred at the lowest clearance. The endplate enhances the air cushion under the lower surface due to blockage of the flow between the endplates. As a result, the $C_{P}$ increased by $14 \%$ and $124 \%$ near the leading edge and the trailing edge, respectively, compared to the case of WOE. Thus, $f$ revealed that the aerodynamic performance of WOE and WWE was maximum at the lowest value, $h / C=0.05$, independent of the endplate. The maximum $f$ of WWE increased $46 \%$ from that of WOE. The difference in the value of $f$ between WOE and WWE became small with increasing $h / C$.

The endplate considerably modifies the formation of tip vortices. The tip vortex of WWE moved more laterally that of WOE that caused a decrease in the induced drag, thus leading to a reduction in the total drag.

\section{ACKNOWLEDGMENTS}

This work was supported by the National Research Foundation of Korea (NRF) grant funded by the Korea Government (MEST) through GCRC-SOP (No. 2011-0030662) and the National Research Foundation of Korea (NRF) grant funded by the Korea Government (MEST) (No. 2010-0025618).

\section{REFERENCES}

Ahmed, N.A. and Goonaratne, J., 2002. Lift augmentation of a low-aspect-ratio thick wing in ground effect. Journal of Aircraft, 39(2), pp.381-384.

Ahmed, M.R. and Sharma, S.D., 2005. An investigation on the aerodynamics of a symmetrical airfoil in ground effect. Experimental Thermal and Fluid Science, 29(6), pp.633-647.

Chun, H.H., Chang, J.H., Paik, K.J. and Chang, S.I., 1997. Preliminary design of a 20 passenger PARWIG craft and construction of a 1/10 scale radio controlled model. Proceeding of International Conference on FAST Sea Transportation. Sydney, Australia, 513-520.

Chun, H.H. and Chang, C.H., 2002. Longitudinal stability and dynamic motions of a small passenger WIG craft. Ocean Engineering, 29(10), pp.1145-1162.

Fink, P.M. and Lastinger, L.J., 1996. Aerodynamics characteristics of Low-Aspect-Ratio wings in close proximity to the ground. Report, TN D-926. USA, NASA.

Fluent, 2009. Fluent user's guide version 12.0.3. Lebanon, ANSYS Inc.

Hirt, C.W. and Nichols, B.D., 1981. Volume of fluid (VOF) method for the dynamics of free boundaries. Journal of Computational Physics, 39, pp.201-225.

Hsiun, C.M. and Chen, C.K., 1995. Numerical investigation of the thickness and camber effects on aerodynamic characteristics for two-dimensional airfoils with ground effect in viscous flow. Transactions of the Japan Society for Aeronautical and Space Sciences, 38(119), pp.77-90.

Hsiun, C.M. and Chen, C.K., 1996. Aerodynamic characteristics of a two-dimensional airfoil with ground effect. Journal of Aircraft, 33(2), pp.386- 392.

Han, C., Cho, L. and Cho, J., 2005. Wake shapes behind wings in close formation flight near the ground. Journal of Mechanical Science and Technology, 19(2), pp.674-681.

Jung, J.H., Yoon, H.S., Chun, H.H., Hung, P.A. and Elsamni, O.A., 2012. Mean flow characteristics of two-dimensional wings in ground effect. International Journal of Architecture and Ocean Engineering, 4(2). pp.151-161.

Kim, S.K., Suh, S.B., Lee, D.H. and Kim, K.E., 1997. Wind tunnel test study on the wings of WIG ship. Journal of the Society of Naval Architects of Korea, 34(1), pp.60-67. 
Kim, Y., Lee, J.E., Shin, M.S., Kang, K.J. and Kwon, J.H., 2006. Turbulent flow simulation on the ground effect about a $2-$ dimensional airfoil. Journal of computational fluids engineering, 11(4), pp.81-89.

Mizutani, N. and Suzuki, K., 1993. Numerical analysis of 3-D WIG advancing over the still water surface. Journal of the Society of Naval Architects of Japan, 174, pp.35-46.

Moon, Y.J., Oh, H.J. and Seo, J.H., 2005. Aerodynamic investigation of three-dimensional wings in ground effect for aerolevitation electric vehicle. Aerospace Science and Technology, 9(6), pp.485-494.

Nuhait, A.O. and Mook, D.T., 1989. Numerical simulation of wings in steady and unsteady ground effects. Journal of Aircraft, 26(12), pp.1081-1089.

Raymond, A.E., 1921. Ground influence on aerofoil. Report, NACA Technical Note 67. USA, Massachusetts Institute of Technology.

Reid, E.G., 1927. A full-scale investigation of ground effect. Report, NACA Technical Report 265. USA, Langley Memorial Aeronautical Laboratory.

Recant, I.R., 1939. Wind-tunnel investigation of ground effect on wings with flap. Report, NACA Technical Note 705. USA, Langley Memorial Aeronautical Laboratory.

Serebrisky, Y.M., Biachuev, S. A., 1946. Wind-tunnel investigation of the horizontal motion of a wing near the ground. Report, NACA Technical Note 1095. USA, Central Aero-Hydrodynamical Institute.

Shin, M.S., Yang, S.I., Joo, Y.R., Kim, S.K., Bae, Y.S., Kim, J.H. and Chun, H.H., 1997. Wind tunnel test results for eight and twenty passenger class WIG effect ships. Proceeding of International Conference on FAST Sea Transportation. Sydney, Australia, 565-570.

Zerihan, J. and Zhang, X., 2000. Aerodynamics of a single element wing in ground effect. Journal of Aircraft, 37(6), pp. 1058-1064.

Zhang, X., Zerihan, J., Ruhrmann, A. and Deviese, M., 2002. Tip vortices generated by a wing in ground effect. Proceedings of the 11th International Symposium on Applications of Laser Techniques to Fluid Mechanics. Lisbon, Portugal. 